\title{
Vibration analysis of rectangular plates with one or more guided edges via bicubic B-spline method
}

\author{
W.J. Si ${ }^{\mathrm{a}}$, K.Y. Lam ${ }^{\mathrm{a}}$ and S.W. Gong ${ }^{\mathrm{b}, *}$ \\ ${ }^{a}$ Department of Mechanical Engineering, National University of Singapore, 10 Kent Ridge Crescent, 119260 , \\ Singapore \\ ${ }^{\mathrm{b}}$ Institute of High Performance of Computing, 1 Science Park Road No. 01-01, the Capricorn, Science Park II, \\ 117528, Singapore
}

Received 19 January 2004

Revised 17 January 2005

\begin{abstract}
A simple and accurate method is proposed for the vibration analysis of rectangular plates with one or more guided edges, in which bicubic B-spline interpolation in combination with a new type of basis cubic B-spline functions is used to approximate the plate deflection. This type of basis cubic B-spline functions can satisfy simply supported, clamped, free, and guided edge conditions with easy numerical manipulation. The frequency characteristic equation is formulated based on classical thin plate theory by performing Hamilton's principle. The present solutions are verified with the analytical ones. Fast convergence, high accuracy and computational efficiency have been demonstrated from the comparisons. Frequency parameters for 13 cases of rectangular plates with at least one guided edge, which are possible by approximate or numerical methods only, are presented. These results are new in literature.
\end{abstract}

Keywords: Cubic B-spline function, bicubic B-spline interpolation, vibration analysis, rectangular plate, guided edge condition

\section{Notation}

$\begin{array}{ll}{\left[A_{x}\right],\left[B_{x}\right],\left[C_{x}\right],\left[F_{x}\right]} & \text { spline matrices; } \\ {\left[A_{y}\right],\left[B_{y}\right],\left[C_{y}\right],\left[F_{y}\right]} & \\ a, b & \text { Plate lengths in } x \text { and } y \text { directions, respectively; } \\ a_{i} & \text { Undetermined spline nodal coefficient of beam; } \\ a_{i, j} & \text { Undetermined spline nodal coefficient of plate; } \\ {[D]} & \text { Rigidity matrix of plate; } \\ d & \text { Plate thickness; } \\ E & \text { Young's moduli of plate material; } \\ {[H],[N]} & \text { Spline interpolation matrices; } \\ h & \text { Spline section length of beam; } \\ h_{x}, h_{y} & \text { Spline section length in } x \text { and } y \text { directions, respectively; } \\ {[K]} & \text { Stiffness matrix; } \\ l & \text { Beam length; } \\ {[M]} & \text { Mass matrix; } \\ N, M & \text { Spline section in } x \text { and } y \text { directions, respectively; }\end{array}$

*Corresponding author. E-mail: gongsw@ihpc.a-star.edu.sg. 


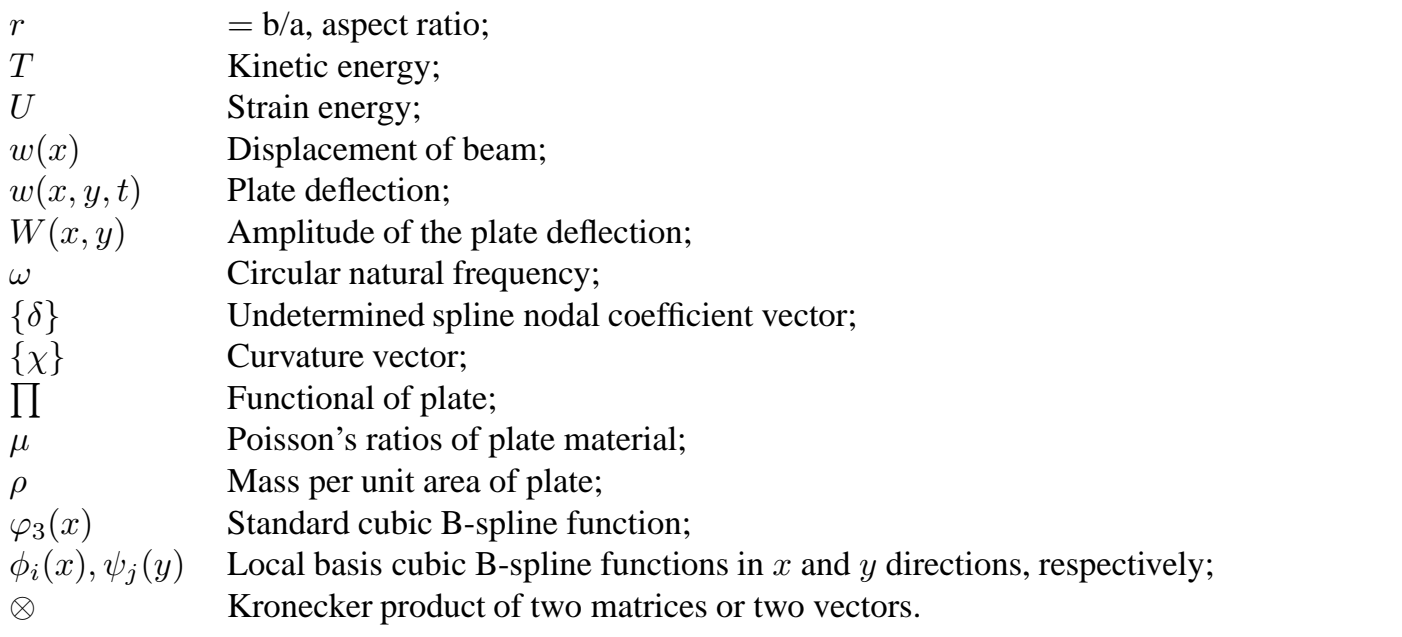

\section{Introduction}

Flexural vibration of rectangular plates under various combinations of the three classical edge conditions, i.e. simply supported, clamped and free edges, has been studied extensively. The three classical edge conditions are simply three of the four possible combinations of essential and natural conditions. The fourth mathematically possible boundary condition has zero rotation (essential condition) and zero effective shear force (natural condition), which has been referred to in the literature as the guided [2] or sliding edge [11]. Although the fourth edge condition is not as important as the three classical ones, it may be encountered in various disciplines of engineering. For example, in mechanical engineering, the bearings may have guided contact with the supported member. The boundary of a piston inside a circular cylinder with narrow clearance may appropriately be modeled as a guided edge.

There exist 21 possible combinations of the three classical edge conditions for rectangular plates, and accurate results for the free vibration of rectangular plates with all 21 combinations of edge conditions have been presented by Leissa [6]. The number of all possible combinations gives rise to 34 additional ones when at least one guided edge is involved. Of all these 34 cases, analytical solution is possible for 21 cases only, and the analytical solutions of the frequency parameters for the 21 cases have been presented by Bert and Malik [2]. The solutions of the remaining 13 cases are possible by approximate or numerical methods only, however, no investigation has been reported. The main purpose of this study is to provide accurate results of frequency parameters for the remaining 13 cases via bicubic B-spline method.

The concept of bicubic spline interpolation was first introduced by Boor [3]. The first application of bicubic B-splines in engineering analysis was reported by Antes [1] for bending analysis of plates. Shen, He and Le [9] presented so-called multivariable spline element method for vibration analysis of rectangular plates. However, their work was limited to plates with clamped or simply supported edges only due to the restrictions of the basis cubic B-spline functions adopted [5].

In this paper, firstly, a new type of basis cubic B-spline functions is constructed. This type of basis cubic B-spline functions can effectively satisfy simply supported, clamped, free and guided edge conditions with easy numerical manipulation. Any beam functions can be accurately approximated by the new type of basis B-spline functions. The plate deflection is chosen as field function and expressed as the product of basis cubic B-spline functions in both $x$ and $y$ directions. The frequency equation is formulated based on classical thin plate theory by performing Hamilton's principle. The validity of the present approach is well established by demonstrating the excellent agreement between the present results and those of analytical solutions by Bert and Malik [2]. As an application of the proposed method, frequency parameters of 13 cases of rectangular plates with at least one guided edge, which are possible by approximate or numerical methods only, are presented. 


\section{Functional of free vibrating plate}

Consider a thin, isotropic rectangular plate of length $a$, width $b$, uniform thickness $d$, and mass per unit area $\rho$. For small amplitude free vibration, the plate deflection $w(x, y, t)$ may be written by

$$
w(x, y, t)=W(x, y) \sin \omega t
$$

where $W(x, y)$ is the amplitude and $\omega$ is the circular natural frequency of the plate. The curvature is given by

$$
\{\chi\}=\left\{\begin{array}{c}
-\partial^{2} W \\
\partial x^{2} \\
\partial^{2} W \\
\partial y^{2} \\
-2 \partial^{2} W \\
\partial x \partial y
\end{array}\right\}
$$

The strain energy $U$ and the kinetic energy $T$ are expressed, respectively, by

$$
\begin{aligned}
& U=\frac{1}{2} \int_{0}^{a} \int_{0}^{b}\{\chi\}^{T}[D]\{\chi\} d y d x \\
& T=\frac{1}{2} \int_{0}^{a} \int_{0}^{b} \rho\left(\begin{array}{c}
\partial w \\
\partial t
\end{array}\right)^{2} d y d x
\end{aligned}
$$

in which $[D]$ is flexural rigidity matrix, as follows:

$$
[D]=\begin{gathered}
E d^{3} \\
12\left(1-\mu^{2}\right)
\end{gathered}\left[\begin{array}{ccc}
1 & \mu & 0 \\
\mu & 1 & 0 \\
0 & 0 & (1-\mu) / 2
\end{array}\right]
$$

where $E$ is Young's modulus, $\mu$ is Poisson's ratio.

The functional of a thin plate for free vibration is:

$$
\Pi=\underset{\omega}{2 \pi} \int_{0}^{a} \int_{0}^{b}\left(\{\chi\}^{T}[D]\{\chi\}-\rho \omega^{2} W^{2}\right) d y d x
$$

\section{Bicubic B-spline interpolation}

The mathematical expression of standard cubic B-spline function can be expressed as

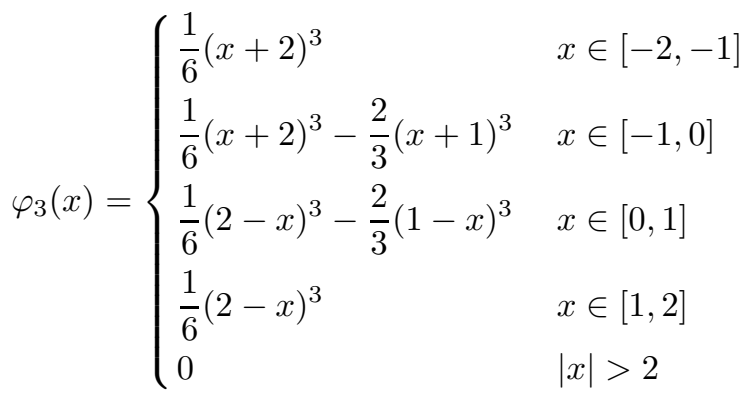

The graphical representation of standard cubic B-spline function is shown in Fig. 1. It's seen from Fig. 1 that standard cubic B-spline function is a piecewise cubic polynomial. Cubic B-spline function has mathematical properties which are advantageous in numerical analysis. The mathematical properties of cubic B-spline functions are well documented by Boor [4] and Schumaker [8].

Consider a uniform beam of length $l$. The beam is divided into $N$ equivalent spline sections by means of spline nodes. The beam deflection can be taken as the summation of $N+3$ terms and each term can be represented by a 


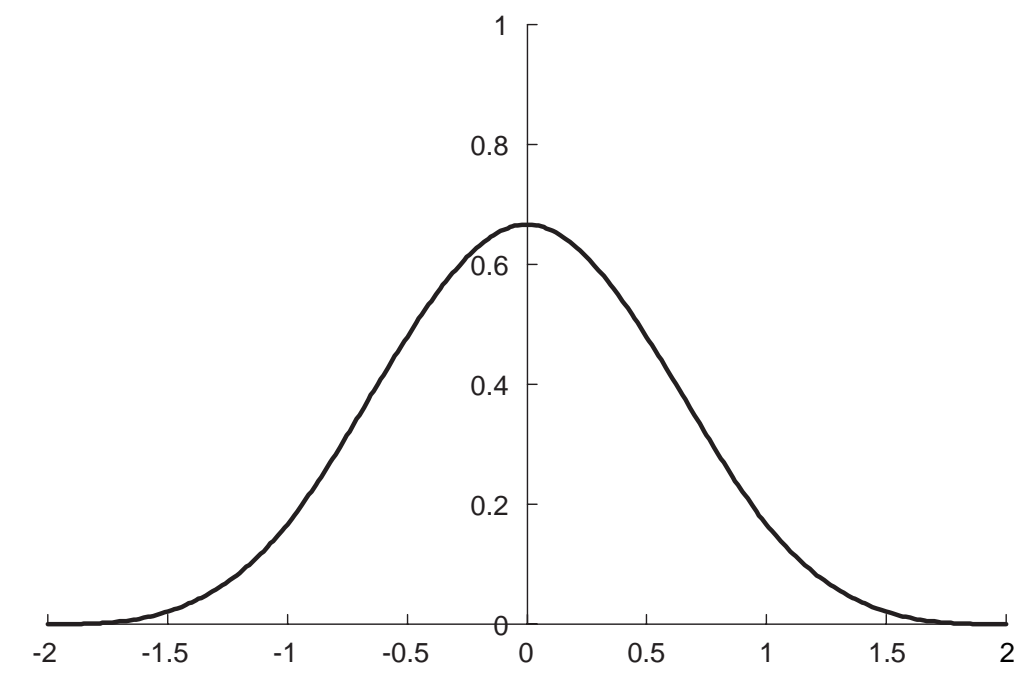

Fig. 1. Standard cubic B-spline function.

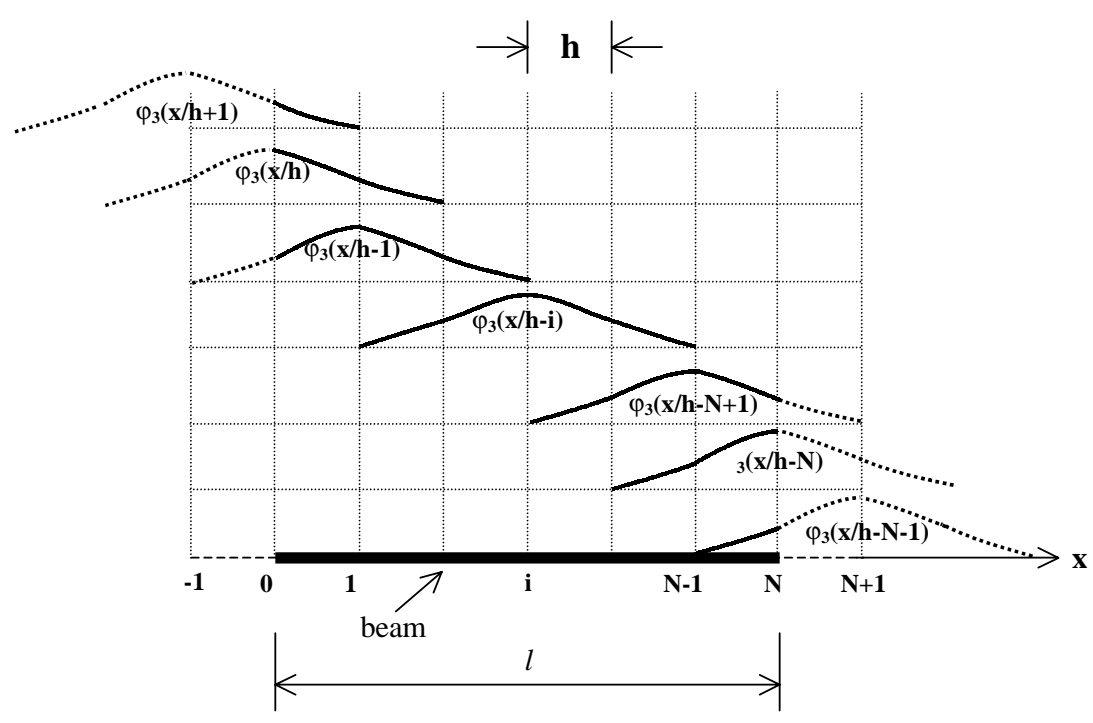

Fig. 2. Beam function interpolation by ordinary cubic B-spline functions.

local dimensionless cubic B-spline function, as shown in Fig. 2. It's observed from Fig. 2 that, at any spline node, the displacement expression has three non-zero terms only, e.g. only $\varphi_{3}\left({ }_{h}^{x}-(i-1)\right), \varphi_{3}\left({ }_{h}^{x}-i\right)$, and $\varphi_{3}\left({ }_{h}^{x}-(i+1)\right)$ have non-zero contributions to the displacement at the $i$ th spline node. However, it's difficult to account for different boundary conditions using ordinary cubic B-spline interpolation. The three local B-spline functions at the boundary, therefore, must be constructed to accommodate different edge conditions. Several kinds of basis cubic B-spline functions have been proposed by Qin [7] and one of which has been commonly used by other researchers $[9,10$, 12]. This commonly used kind of basis cubic B-spline functions can satisfy simply supported and clamped edges conveniently, however, free and guided edges may not be satisfied [5].

\subsection{The new type of basis cubic B-spline functions}

In the present paper, a new type of basis cubic B-spline functions is constructed, as follows 


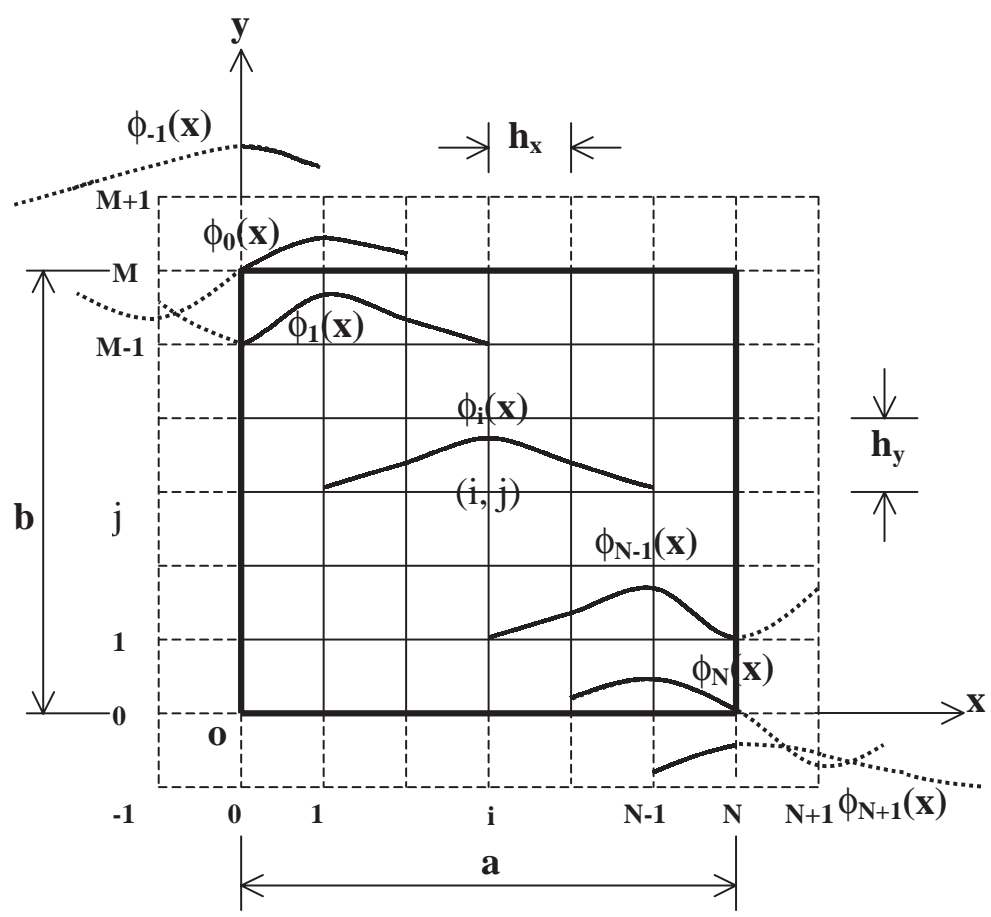

Fig. 3. Plate deflection interpolation by basis cubic B-spline functions ( $x$-direction).

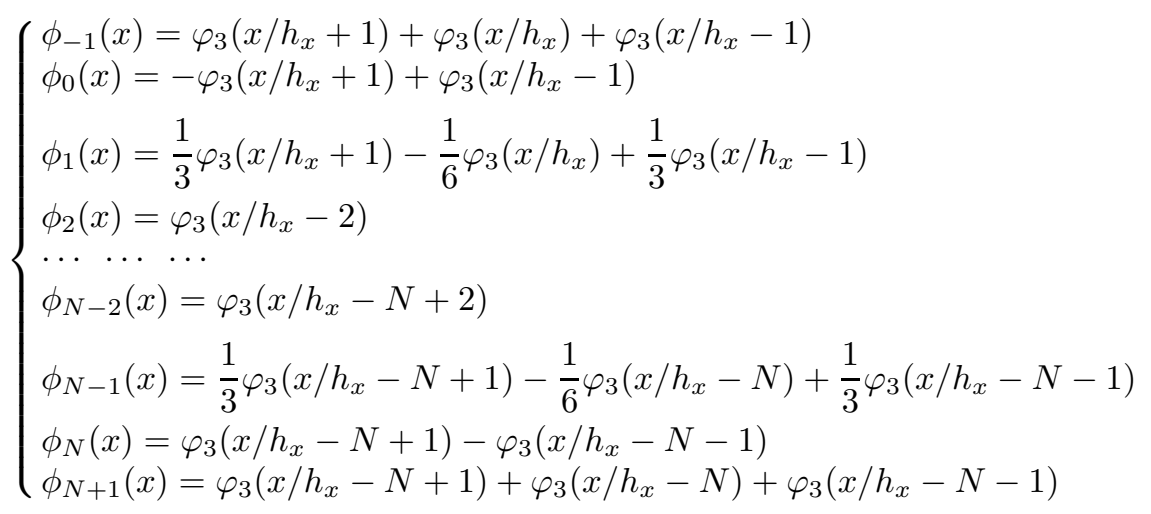

The three boundary cubic B-spline functions at both ends are linearly combined to satisfy different edge conditions, as sketched in Fig. 3. By virtue of the new type of basis cubic B-spline functions in Eq. (8), the beam deflection can be expressed as

$$
w(x)=\sum_{i=-1}^{N+1} \phi_{i}(x) a_{i}
$$

where $a_{i}(i=-1,0,1, \ldots, \mathrm{N}-1, \mathrm{~N}, \mathrm{~N}+1)$ is undetermined coefficient at the $i$ th spline node.

It's well known that in energy approaches admissible functions have to satisfy geometrical boundary conditions but need not satisfy natural boundary conditions. However, from a practical consideration of the rate of convergence, it is desirable to satisfy natural boundary conditions if possible [13]. The new type of basis cubic B-spline functions has desirable characteristics for numerical analysis since it can satisfy all geometrical boundary conditions and part of natural boundary conditions of the four kinds of edge conditions. For example, at the end $x=0$, Eq. (9) yields

$$
w(0)=a_{-1} \phi_{-1}(0)+a_{0} \phi_{0}(0)+a_{1} \phi_{1}(0)
$$


Table 1

Convergence study of bicubic B-spline solutions of frequency parameters for SSSG square plates

\begin{tabular}{ccccccc} 
Num. of & \multicolumn{5}{c}{ Frequency parameters } \\
sections & 1st. & 2nd. & 3rd. & 4th. & 5th. & 6th. \\
$6 \times 6$ & 12.33745 & 32.08115 & 41.98588 & 61.71575 & 71.71681 & 91.92226 \\
$8 \times 8$ & 12.33714 & 32.07767 & 41.95718 & 61.69363 & 71.59787 & 91.45260 \\
$10 \times 10$ & 12.33706 & 32.07679 & 41.95024 & 61.68836 & 71.57095 & 91.35205 \\
$12 \times 12$ & 12.33703 & 32.07649 & 41.94789 & 61.68658 & 71.56216 & 91.32023 \\
$14 \times 14$ & 12.33701 & 32.07636 & 41.94692 & 61.68585 & 71.55858 & 91.30756 \\
$16 \times 16$ & 12.33701 & 32.07630 & 41.94646 & 61.68551 & 71.55691 & 91.30168 \\
$18 \times 18$ & 12.33701 & 32.07627 & 41.94621 & 61.68532 & 71.55604 & 91.29865 \\
$20 \times 20$ & 12.33701 & 32.07625 & 41.94608 & 61.68522 & 71.55554 & 91.29696 \\
Exact* & 12.33701 & 32.07622 & 41.94582 & 61.68503 & 71.55464 & 91.29385
\end{tabular}

${ }^{*}$ Bert and Malik [2].

Table 2

Comparison of frequency parameters for SCSG plates

\begin{tabular}{crcccrr} 
Mode & \multicolumn{2}{c}{$r=0.4$} & \multicolumn{2}{c}{$r=1.0$} & \multicolumn{2}{c}{$r=2.5$} \\
sequence & Present & Exact* & Present & \multicolumn{1}{c}{ Exact* } & Present & \multicolumn{1}{c}{ Exact* } \\
1 & 10.34459 & 10.34454 & 13.68579 & 13.68577 & 41.18469 & 41.18466 \\
2 & 14.04479 & 14.04455 & 38.69439 & 38.69393 & 65.27708 & 65.27632 \\
3 & 21.15134 & 21.15106 & 42.58779 & 42.58662 & 111.25348 & 111.24204 \\
4 & 31.48613 & 31.47916 & 66.30066 & 66.29910 & 178.53572 & 178.45914 \\
5 & 39.91245 & 39.91111 & 83.49563 & 83.48830 & 197.06605 & 197.06296 \\
6 & 43.36284 & 43.36022 & 91.71810 & 91.70417 & 222.30722 & 222.30413 \\
7 & 45.00561 & 44.97255 & 111.01629 & 111.00916 & 266.23690 & 266.16636 \\
8 & 50.20771 & 50.20251 & 114.37324 & 114.35987 & 266.50405 & 266.22905 \\
9 & 60.37257 & 60.36157 & 147.92791 & 147.87515 & 330.11816 & 330.07159 \\
* Bert and Malik [2]. & & & & &
\end{tabular}

And at the end $x=0$, Eq. (8) gives

$$
\begin{aligned}
\phi_{-1}(0) & =1, \phi_{i}(0)=0(i=0,1,2, \cdots, N+1) \\
\phi_{0}^{\prime}(0) & =1, \phi_{i}^{\prime}(0)=0(i=-1,1,2, \cdots, N+1) \\
\phi^{\prime \prime}{ }_{1}(0) & =1, \phi^{\prime \prime}{ }_{i}(0)=0(i=-1,0,2, \cdots, N+1)
\end{aligned}
$$

It's observed from Eqs (10-13) that only one term has contribution to the deflection, slope and second derivative of the deflection at the boundary. Therefore, except the prescribed values for third derivatives of the deflection at the boundary, the new type of basis cubic B-spline functions can satisfy geometrical boundary conditions and prescribed second derivative of deflection for the four kinds of edge conditions.

For example, for clamped end of the beam at $x=0$, the boundary conditions are $w(0)=0, w^{\prime}(0)=0$ and $w^{\prime \prime}(0) \neq 0$. Those undetermined coefficients corresponding to $\phi_{-1}(0)$ and $\phi_{0}(0)$, i.e. $a_{-1}$ and $a_{0}$, thus should be equal to zero. For simply supported end of the beam at $x=0$, the boundary condition is $w(0)=0, w^{\prime}(0) \neq$ $0, w^{\prime \prime}(0)=0$, thus $a_{-1}$ and $a_{1}$ should be equal to zero. For free end of the beam at $x=0$, the boundary condition is $w(0) \neq 0, w^{\prime}(0) \neq 0, w^{\prime \prime}(0)=0$, thus $a_{1}$ should vanish. Likewise, for guided end of the beam at $x=0$, the boundary condition is $w(0) \neq 0, w^{\prime}(0)=0, w^{\prime \prime}(0) \neq 0$, thus $a_{0}$ should vanish. The case for the end at $a=l$ is similar. Hence, simply supported, clamped, free and guided edges can be implemented with ease by using the new type of basis cubic B-spline functions. It should be noted that the numerical manipulation to eliminate the corresponding coefficients can be easily carried out in computer programme.

\subsection{Bicubic B-spline interpolation}

To express the plate deflection by bicubic B-spline approximation, the plate is divided into $N$ and $M$ equivalent spline sections in $x$ and $y$ directions, respectively, as shown in Fig. 3. Thus 
Table 3

Comparison of frequency parameters for SGGF plates

\begin{tabular}{crcrcrr} 
Mode & \multicolumn{2}{c}{$r=0.4$} & \multicolumn{2}{c}{$r=1.0$} & \multicolumn{2}{c}{$r=2.5$} \\
sequence & \multicolumn{1}{c}{ Present } & Exact ${ }^{*}$ & Present & Exact* & Present & \multicolumn{1}{c}{ Exact* } \\
1 & 2.44325 & 2.44001 & 2.41070 & 2.40785 & 2.37357 & 2.37104 \\
2 & 3.77012 & 3.76564 & 9.18348 & 9.18141 & 21.76868 & 21.74211 \\
3 & 7.79626 & 7.79427 & 22.02673 & 21.99667 & 39.03164 & 39.03119 \\
4 & 14.82359 & 14.82200 & 30.55345 & 30.51000 & 60.93475 & 60.85150 \\
5 & 22.14358 & 22.10998 & 33.42682 & 33.42615 & 65.94330 & 65.92023 \\
6 & 23.60167 & 23.55383 & 56.21210 & 56.18961 & 110.63301 & 110.53142 \\
7 & 25.01826 & 25.00849 & 61.40349 & 61.31043 & 119.88639 & 119.68813 \\
8 & 28.09377 & 28.05593 & 70.35809 & 70.11123 & 172.64695 & 172.39071 \\
9 & 35.52962 & 35.50419 & 77.61083 & 77.60132 & 192.17749 & 192.17367
\end{tabular}

* Bert and Malik [2].

$$
\begin{aligned}
& 0=x_{0}<x_{1}<\cdots<x_{i}<\cdots<x_{N}=a, x_{i}=x_{0}+i h_{x}, h_{x}=a / N \\
& 0=y_{0}<y_{1}<\cdots<y_{i}<\cdots<y_{M}=b, y_{i}=y_{0}+i h_{y}, h_{y}=b / M
\end{aligned}
$$

where $N$ and $M$ are integers and $N, M>4, h_{x}$ and $h_{y}$ are spline section lengths in $x$ and $y$ directions, respectively.

Based on the technique of separation of variables, the amplitude of the plate deflection may be represented in the form

$$
W(x, y)=\sum_{i=-1}^{N+1} \sum_{j=-1}^{M+1} \psi_{j}(y) \phi_{i}(x) a_{i, j}
$$

where $a_{i, j}$ is undetermined coefficient at the spline node $(i, j), \phi_{i}(x)$ and $\psi_{j}(y)$ are the $i$ th and $j$ th local basis cubic B-spline functions in $x$ and $y$ directions, respectively, as in Eq. (8). In matrix form, Eq. (14) can be written as

$$
W(x, y)=[\psi(y)] \otimes[\phi(x)]\{\delta\}
$$

where $[\phi(x)]$ and $[\psi(y)]$ are basis cubic B-spline functions vectors in $x$ and $y$ directions, respectively, as follows

$$
\begin{aligned}
& {[\phi(x)]=\left[\phi_{-1}(x) \phi_{0}(x) \phi_{1}(x) \phi_{2}(x) \cdots \phi_{N-1}(x) \phi_{N}(x) \phi_{N+1}(x)\right]} \\
& {[\psi(y)]=\left[\psi_{-1}(y) \psi_{0}(y) \psi_{1}(y) \psi_{2}(y) \cdots \psi_{M-1}(y) \psi_{M}(y) \psi_{M+1}(y)\right]}
\end{aligned}
$$

and

$$
\{\delta\}=\left\{\{a\}_{-1}^{T}\{a\}_{0}^{T}\{a\}_{1}^{T} \cdots\{a\}_{M}^{T}\{a\}_{M+1}^{T}\right\}^{T}
$$

is undetermined spline nodal coefficient vector, in which

$$
\{a\}_{j}=\left\{a_{-1, j} a_{0, j} a_{1, j} \cdots a_{N, j} a_{N+1, j}\right\}^{T}, j=-1,0,1, \cdots, M, M+1
$$

and the symbol, $\otimes$, means the Kronecker product of two matrices or two vectors.

\section{Frequency characteristic equation}

The amplitude of the plate deflection in Eq. (15) can be further written as

$$
W(x, y)=[\psi(y)] \otimes[\phi(x)]\{\delta\}=[N]\{\delta\}
$$

The curvature vector in Eq. (2) can, therefore, be expressed as

$$
\{\chi\}=\left\{\begin{array}{c}
-\partial^{2} W \\
\partial x^{2} \\
\partial^{2} W \\
\partial y^{2} \\
\partial^{2} W \\
\partial x \partial y
\end{array}\right\}=[H]\{\delta\}
$$


Table 4

Comparison of frequency parameters for GGGG plates

\begin{tabular}{cccrrrr}
$\begin{array}{c}\text { Mode } \\
\text { sequence }\end{array}$ & \multicolumn{2}{c}{$r=0.4$} & \multicolumn{2}{c}{$r=1.0$} & \multicolumn{2}{c}{$r=2.5$} \\
1 & 1.57914 & 1.57914 & \multicolumn{1}{c}{ Present } & \multicolumn{1}{c}{ Exact* } & \multicolumn{1}{c}{ Present } & \multicolumn{1}{l}{ Exact* } \\
2 & 6.31673 & 6.31655 & 19.73923 & 19.86960 & 9.86962 & 9.869620 \\
3 & 9.86962 & 9.86960 & 39.47958 & 39.47842 & 39.47958 & 39.47842 \\
4 & 11.44876 & 11.44874 & 49.34896 & 49.34802 & 61.68514 & 61.68503 \\
5 & 14.21448 & 14.21223 & 78.95801 & 78.95684 & 88.55473 & 71.55464 \\
6 & 16.18624 & 16.18615 & 88.84053 & 88.82644 & 101.16397 & 101.82644 \\
7 & 24.08319 & 24.08184 & 98.70876 & 98.69605 & 150.51997 & 150.51148 \\
8 & 25.27991 & 25.26619 & 128.31508 & 128.30486 & 157.99945 & 157.91368 \\
9 & 35.14584 & 35.13579 & 157.99945 & 157.91368 & 219.66152 & 219.59871
\end{tabular}

Table 5

Bicubic B-spline solutions of frequency parameters for CCCG plates

\begin{tabular}{cccccr} 
Mode & \multicolumn{5}{c}{ Aspect ratio $r=\mathrm{a} / \mathrm{b}$} \\
sequence & \multicolumn{6}{c}{$2 / 5$} & $2 / 3$ & \multicolumn{1}{c}{1} & \multicolumn{1}{c}{$3 / 2$} & \multicolumn{1}{c}{$5 / 2$} \\
1 & 22.63272 & 23.19655 & 24.57795 & 28.70100 & 46.70085 \\
2 & 24.86870 & 30.74236 & 44.77075 & 67.54214 & 82.05066 \\
3 & 29.97003 & 47.96869 & 63.98760 & 80.19072 & 139.49409 \\
4 & 38.50371 & 62.62624 & 83.27823 & 115.82573 & 199.23378 \\
5 & 50.64564 & 70.44395 & 87.26174 & 126.64734 & 217.67627 \\
6 & 61.99886 & 75.06327 & 123.28343 & 173.24566 & 230.55915 \\
7 & 64.60543 & 86.90657 & 123.69770 & 179.13252 & 283.30350 \\
8 & 66.37193 & 111.59241 & 142.40142 & 205.67903 & 316.34459 \\
9 & 69.97816 & 112.67860 & 150.60841 & 212.52122 & 357.55418
\end{tabular}

Table 6

Bicubic B-spline solutions of frequency parameters for CCSG plates

\begin{tabular}{cccccc} 
Mode & \multicolumn{5}{c}{ Aspect ratio $r=\mathrm{a} / \mathrm{b}$} \\
sequence & $2 / 5$ & $2 / 3$ & 1 & $3 / 2$ & \multicolumn{1}{c}{$5 / 2$} \\
1 & 15.77075 & 16.53117 & 18.34855 & 23.45847 & 43.44845 \\
2 & 18.69489 & 25.78157 & 41.25078 & 56.70824 & 72.92613 \\
3 & 24.86585 & 44.61565 & 52.63283 & 77.98472 & 124.64896 \\
4 & 34.44482 & 51.06220 & 74.08709 & 108.68130 & 197.38366 \\
5 & 47.41460 & 59.93062 & 85.15311 & 110.58450 & 198.09013 \\
6 & 50.33792 & 72.68709 & 106.86083 & 160.52812 & 226.12866 \\
7 & 53.32496 & 77.97819 & 116.82442 & 177.82731 & 274.27814 \\
8 & 59.39298 & 105.28626 & 127.68015 & 184.49038 & 290.73972 \\
9 & 63.75092 & 105.37240 & 149.14356 & 207.77138 & 343.21661
\end{tabular}

Thus we have

$$
\begin{aligned}
& {[N]=[\psi(y)] \otimes[\phi(x)]} \\
& {[H]=\left\{\begin{array}{l}
-[\psi(y)] \otimes\left[\phi^{\prime \prime}(x)\right] \\
-\left[\psi^{\prime \prime}(y)\right] \otimes[\phi(x)] \\
-2\left[\psi^{\prime}(y)\right] \otimes\left[\phi^{\prime}(x)\right]
\end{array}\right\}}
\end{aligned}
$$

Upon substitution of Eqs (19) and (20) into Eq. (6), the following expression is obtained

$$
\Pi={ }_{\omega}^{2 \pi}\{\delta\}^{T}\left([K]-\omega^{2}[M]\right)\{\delta\}
$$

where $[K]$ and $[M]$ are the stiffness matrix and the mass matrix of the plate, respectively, as follows

$$
[K]=\int_{0}^{a} \int_{0}^{b}[H]^{T}[D][H] d y d x
$$


Table 7

Bicubic B-spline solutions of frequency parameters for CCGF plates

\begin{tabular}{ccrrrr} 
Mode & \multicolumn{5}{c}{ Aspect ratio $r=\mathrm{a} / \mathrm{b}$} \\
sequence & \multicolumn{1}{c}{$2 / 5$} & $2 / 3$ & \multicolumn{1}{c}{1} & \multicolumn{1}{c}{$3 / 2$} & \multicolumn{1}{c}{$5 / 2$} \\
1 & 5.82781 & 6.39955 & 7.78816 & 11.52535 & 24.93200 \\
2 & 8.25672 & 13.98630 & 25.85303 & 35.53868 & 47.46232 \\
3 & 13.87844 & 30.89554 & 32.29520 & 53.12424 & 90.53861 \\
4 & 22.92983 & 31.03902 & 51.23587 & 78.60378 & 141.03696 \\
5 & 30.46421 & 39.20468 & 64.92146 & 79.56457 & 153.69023 \\
6 & 33.27805 & 55.74725 & 76.53554 & 123.99143 & 166.47386 \\
7 & 35.33239 & 56.95635 & 89.29651 & 141.87340 & 213.48687 \\
8 & 39.00428 & 75.36847 & 95.95572 & 143.37222 & 236.93056 \\
9 & 47.78803 & 81.08415 & 123.94247 & 165.66525 & 279.53734
\end{tabular}

Table 8

Bicubic B-spline solutions of frequency parameters for CCGG plates

\begin{tabular}{ccrcrr} 
Mode & \multicolumn{5}{c}{ Aspect ratio $r=\mathrm{a} / \mathrm{b}$} \\
sequence & $2 / 5$ & $2 / 3$ & 1 & \multicolumn{1}{c}{$3 / 2$} & \multicolumn{1}{c}{$5 / 2$} \\
1 & 5.91096 & 6.75124 & 8.99631 & 15.19030 & 36.94347 \\
2 & 8.85439 & 16.63070 & 32.89572 & 37.41907 & 55.33993 \\
3 & 15.37489 & 31.32443 & 33.05174 & 70.47996 & 96.09306 \\
4 & 25.35216 & 36.05379 & 55.00942 & 81.12103 & 158.45099 \\
5 & 30.59011 & 40.30721 & 77.23363 & 90.69122 & 191.18820 \\
6 & 33.54399 & 58.66116 & 77.29907 & 131.98762 & 209.64994 \\
7 & 38.64062 & 64.45888 & 98.19975 & 145.03248 & 241.50389 \\
8 & 39.62308 & 75.74074 & 98.48365 & 170.41666 & 247.64425 \\
9 & 48.98082 & 84.55412 & 140.54532 & 190.24676 & 306.13010
\end{tabular}

$[M]=\int_{0}^{a} \int_{0}^{b} \rho[N]^{T}[N] d y d x$

By Hamilton's principle, the functional $\Pi$ in Eq. (23) can be minimized with respect to the undetermined coefficient vector $\{\delta\}$. The minimization procedure results in the generalized eigenvalue equation as follows

$$
\left([K]-\omega^{2}[M]\right)\{\delta\}=0
$$

With the definition of the following spline matrices

$$
\begin{aligned}
& {\left[A_{x}\right]=\int_{0}^{a}\left[\phi^{\prime \prime}(x)\right]^{T}\left[\phi^{\prime \prime}(x] d x\right.} \\
& {\left[B_{x}\right]=\int_{0}^{a}[\phi(x)]^{T}\left[\phi^{\prime \prime}(x] d x\right.} \\
& {\left[C_{x}\right]=\int_{0}^{a}\left[\phi^{\prime}(x)\right]^{T}\left[\phi^{\prime}(x] d x\right.} \\
& {\left[F_{x}\right]=\int_{0}^{a}[\phi(x)]^{T}[\phi(x] d x}
\end{aligned}
$$

Equations (24) and (25) can be written as

$$
\begin{aligned}
{[K]=} & \begin{aligned}
E d^{3} \\
12\left(1-\mu^{2}\right)
\end{aligned}\left(\left[F_{y}\right] \otimes\left[A_{x}\right]+\left[A_{y}\right] \otimes\left[F_{x}\right]+\mu\left[B_{y}\right]^{T} \otimes\left[B_{x}\right]+\mu\left[B_{y}\right] \otimes\left[B_{x}\right]^{T}\right. \\
& \left.+2(1-\mu)\left[C_{y}\right] \otimes\left[C_{x}\right]\right) \\
{[M]=} & \rho\left[F_{y}\right] \otimes\left[F_{x}\right]
\end{aligned}
$$


Table 9

Bicubic B-spline solutions of frequency parameters for CGCF plates

\begin{tabular}{ccccrr} 
Mode & \multicolumn{5}{c}{ Aspect ratio $r=\mathrm{a} / \mathrm{b}$} \\
sequence & $2 / 5$ & $2 / 3$ & 1 & \multicolumn{1}{c}{$3 / 2$} & \multicolumn{1}{c}{$5 / 2$} \\
1 & 22.34398 & 22.31794 & 22.28514 & 22.23789 & 22.15524 \\
2 & 23.16445 & 24.62271 & 27.53553 & 34.19715 & 55.85023 \\
3 & 25.98568 & 33.21762 & 48.55258 & 61.36610 & 61.14986 \\
4 & 31.42507 & 50.46883 & 61.47959 & 76.53181 & 101.62957 \\
5 & 40.05130 & 61.55633 & 68.31690 & 84.88648 & 120.12019 \\
6 & 52.13970 & 64.61253 & 90.30401 & 120.46857 & 165.04976 \\
7 & 61.61644 & 74.50666 & 90.89954 & 128.07138 & 198.93900 \\
8 & 62.71886 & 77.23182 & 120.64817 & 137.17803 & 204.37870 \\
9 & 66.23537 & 92.06798 & 128.15189 & 182.36948 & 246.49511
\end{tabular}

Table 10

Bicubic B-spline solutions of frequency parameters for CGSF plates

\begin{tabular}{cccccc} 
Mode & \multicolumn{5}{c}{ Aspect ratio $r=\mathrm{a} / \mathrm{b}$} \\
sequence & $2 / 5$ & $2 / 3$ & 1 & \multicolumn{1}{c}{$3 / 2$} & \multicolumn{1}{c}{$5 / 2$} \\
1 & 15.38026 & 15.34938 & 15.31182 & 15.25870 & 15.16871 \\
2 & 16.47848 & 18.36153 & 21.92477 & 29.54055 & 49.40109 \\
3 & 20.06276 & 28.49989 & 45.06977 & 49.63005 & 52.58593 \\
4 & 26.48754 & 47.12309 & 49.75065 & 66.56775 & 93.34186 \\
5 & 36.05374 & 49.83304 & 57.50045 & 82.53528 & 103.40695 \\
6 & 48.90650 & 53.32429 & 82.09721 & 103.77033 & 151.55991 \\
7 & 49.89882 & 64.37345 & 88.09754 & 121.01804 & 177.24671 \\
8 & 51.16345 & 74.79419 & 103.95881 & 121.97817 & 203.01177 \\
9 & 55.16032 & 83.37999 & 112.18399 & 177.74621 & 227.91396
\end{tabular}

Table 11

Bicubic B-spline solutions of frequency parameters for CSGF plates

\begin{tabular}{ccrrrr} 
Mode & \multicolumn{5}{c}{ Aspect ratio $r=\mathrm{a} / \mathrm{b}$} \\
sequence & $2 / 5$ & $2 / 3$ & 1 & \multicolumn{1}{c}{$3 / 2$} & \multicolumn{1}{c}{$5 / 2$} \\
1 & 5.75830 & 6.05969 & 6.61272 & 7.70546 & 10.39906 \\
2 & 7.65028 & 11.69383 & 19.95748 & 33.68319 & 39.29193 \\
3 & 12.42087 & 26.15263 & 31.74735 & 38.96296 & 84.96153 \\
4 & 20.57671 & 30.86898 & 47.07998 & 67.27730 & 100.38533 \\
5 & 30.43234 & 37.80435 & 53.63521 & 78.32008 & 129.82887 \\
6 & 32.10642 & 49.83552 & 76.18947 & 114.79794 & 149.52489 \\
7 & 32.95762 & 52.39028 & 80.10300 & 115.91148 & 181.24692 \\
8 & 38.15401 & 75.27012 & 92.85304 & 142.32923 & 233.63307 \\
9 & 46.23710 & 75.40192 & 107.56692 & 142.49455 & 251.08769
\end{tabular}

where $\left[A_{y}\right],\left[B_{y}\right],\left[C_{y}\right],\left[F_{y}\right]$ are the spline matrices similar to $\left[A_{x}\right],\left[B_{x}\right],\left[C_{x}\right]$ and $\left[F_{x}\right]$, but in the $y$ direction. The dimension of the spline matrices $\left[A_{x}\right],\left[B_{x}\right],\left[C_{x}\right]$ and $\left[F_{x}\right]$ is $(N+3) \times(N+3)$ and the dimension of spline matrices $\left[A_{y}\right],\left[B_{y}\right],\left[C_{y}\right]$ and $\left[F_{y}\right]$ is $(M+3) \times(M+3)$. The spline matrices are given in Appendix.

Edge conditions can be imposed by eliminating rows and columns of the spline matrices associated with zero spline nodal coefficients at the boundaries of the plate. For example, for a clamped edge at $x=0$, as stated earlier in last section, the spline nodal coefficients $a_{-1, j}$ and $a_{0, j}(j=-1,0,1, \cdots, \mathrm{M}, \mathrm{M}+1)$ should be equal to zero. Accordingly, the first two rows and columns of the spline matrices $\left[A_{x}\right],\left[B_{x}\right],\left[C_{x}\right]$ and $\left[F_{x}\right]$ should be eliminated.

Similarly, for a simply supported edge at $x=0$, the first and third rows and columns of the spline matrices should be eliminated; and for a free edge at $x=0$, the third row and column of the spline matrices should be eliminated. Likewise, for a guided edge at $x=0$, the second row and column of the spline matrices should be eliminated. The case for the end at $x=a, y=0, y=b$ is similar. Upon application of edge conditions, the free vibration problem of the rectangular plate is reduced to generalized eigenvalue problem in Eq. (26). 
Table 12

Bicubic B-spline solutions of frequency parameters for CGGF plates

\begin{tabular}{ccrrrr} 
Mode & \multicolumn{5}{c}{ Aspect ratio $r=\mathrm{a} / \mathrm{b}$} \\
sequence & \multicolumn{1}{c}{$2 / 5$} & \multicolumn{1}{c}{$2 / 3$} & \multicolumn{1}{c}{1} & \multicolumn{1}{c}{$3 / 2$} & \multicolumn{1}{c}{$5 / 2$} \\
1 & 5.57607 & 5.56300 & 5.54792 & 5.52898 & 5.50107 \\
2 & 6.41026 & 7.91727 & 10.90588 & 17.72148 & 29.81596 \\
3 & 9.62376 & 17.75265 & 30.06285 & 29.97633 & 39.83580 \\
4 & 16.07221 & 30.12290 & 34.22535 & 45.84235 & 70.73926 \\
5 & 25.92440 & 33.43747 & 37.38449 & 71.77492 & 73.93128 \\
6 & 30.17148 & 36.85899 & 61.21624 & 74.22668 & 120.24959 \\
7 & 31.37563 & 44.08551 & 74.38073 & 91.89574 & 137.83999 \\
8 & 35.20760 & 62.80136 & 78.09442 & 99.22643 & 187.40569 \\
9 & 39.11793 & 65.07106 & 82.41132 & 138.27153 & 192.48870
\end{tabular}

Table 13

Bicubic B-spline solutions of frequency parameters for CFGF plates

\begin{tabular}{cccccr} 
Mode & \multicolumn{5}{c}{ Aspect ratio $r=\mathrm{a} / \mathrm{b}$} \\
sequence & \multicolumn{1}{c}{$2 / 5$} & \multicolumn{1}{c}{$2 / 3$} & \multicolumn{1}{c}{1} & \multicolumn{1}{c}{$3 / 2$} & \multicolumn{1}{c}{$5 / 2$} \\
1 & 5.56171 & 5.54016 & 5.51922 & 5.49518 & 5.46053 \\
2 & 6.26823 & 7.32162 & 9.00242 & 11.87375 & 18.03545 \\
3 & 8.96880 & 15.13892 & 27.36397 & 29.77589 & 29.55495 \\
4 & 14.57315 & 30.03512 & 29.92623 & 42.76309 & 58.71603 \\
5 & 23.51690 & 31.75483 & 36.29256 & 55.13088 & 73.36098 \\
6 & 30.12570 & 33.01511 & 57.04120 & 73.86955 & 111.28386 \\
7 & 31.22434 & 42.73659 & 65.79679 & 87.00912 & 136.99304 \\
8 & 34.87247 & 57.58303 & 74.15486 & 89.40988 & 144.42500 \\
9 & 35.82138 & 59.73655 & 81.41000 & 137.78089 & 178.21389
\end{tabular}

Table 14

Bicubic B-spline solutions of frequency parameters for SGFF plates

\begin{tabular}{cccccc} 
Mode & \multicolumn{5}{c}{ aspect ratio $r=\mathrm{a} / \mathrm{b}$} \\
sequence & $2 / 5$ & $2 / 3$ & 1 & $3 / 2$ & $5 / 2$ \\
1 & 2.833583 & 5.153985 & 8.710524 & 14.44969 & 14.92445 \\
2 & 7.197001 & 15.03607 & 15.29102 & 16.77704 & 38.26339 \\
3 & 14.13896 & 16.25319 & 16.39972 & 37.02406 & 49.48403 \\
4 & 15.43609 & 20.89188 & 32.93346 & 49.50993 & 64.47378 \\
5 & 17.51225 & 34.45655 & 49.63856 & 69.95977 & 102.9047 \\
6 & 23.17969 & 35.70995 & 53.91768 & 73.52203 & 106.1977 \\
7 & 24.56755 & 49.77296 & 60.83301 & 93.63335 & 162.7142 \\
8 & 31.83681 & 54.58633 & 76.89855 & 103.8243 & 176.6324 \\
9 & 37.78603 & 55.81457 & 91.10221 & 126.9152 & 191.6661
\end{tabular}

\section{Numerical results}

Based on the bicubic B-spline method presented in the foregoing sections, a general unified computational program is developed for vibration analysis of rectangular plates with one or more guided edges. For the purpose of description, a notation will be adopted as follows. Consider the rectangular plate shown in Fig. 3, the symbolism FSGC, for example, will identify a rectangular plate with edges $x=0, y=0, x=a, y=b$ having free( $\mathrm{F})$, simply supported(S), guided(G), and clamped(C) edge conditions, respectively.

\subsection{Convergence study}

Before making detailed computations it is considered imperative to decide the suitable number of spline sections used and investigate the accuracy of the solutions. The rectangular SSSG plate is used for convergence study. The exact solutions of the dimensionless natural frequency $\Omega=\omega a^{2} \sqrt{ } \rho / D$. are given by Bert and Malik [2]. These results are used to check the accuracy of the results obtained from the present method and determine the suitable number of spline sections. 
Table 15

Bicubic B-spline solutions of frequency parameters for CGFF plates

\begin{tabular}{cccccc} 
Mode & \multicolumn{5}{c}{ Aspect ratio $r=\mathrm{a} / \mathrm{b}$} \\
sequence & $2 / 5$ & $2 / 3$ & 1 & \multicolumn{1}{c}{$3 / 2$} & \multicolumn{1}{c}{$5 / 2$} \\
1 & 3.50985 & 3.50385 & 3.49611 & 3.48488 & 3.46558 \\
2 & 4.83473 & 6.84778 & 10.19646 & 16.97070 & 21.48501 \\
3 & 8.61754 & 16.95434 & 21.86353 & 22.05229 & 39.27830 \\
4 & 15.24552 & 21.98069 & 31.48405 & 42.08476 & 60.95759 \\
5 & 21.95659 & 26.73682 & 34.08862 & 61.11062 & 69.00911 \\
6 & 23.65443 & 36.08935 & 58.15338 & 71.06789 & 114.27546 \\
7 & 25.30255 & 39.78884 & 61.49358 & 82.81370 & 120.13440 \\
8 & 29.00165 & 59.63750 & 71.25972 & 97.34350 & 175.95907 \\
9 & 36.85770 & 61.43003 & 77.55365 & 120.40683 & 190.98657
\end{tabular}

Table 16

Bicubic B-spline solutions of frequency parameters for GGFF plates

\begin{tabular}{ccrrrr} 
Mode & \multicolumn{5}{c}{ Aspect ratio $r=\mathrm{a} / \mathrm{b}$} \\
sequence & $2 / 5$ & $2 / 3$ & 1 & \multicolumn{1}{c}{$3 / 2$} & \multicolumn{1}{c}{$5 / 2$} \\
1 & 0.85908 & 2.38190 & 4.90505 & 5.35928 & 5.36923 \\
2 & 4.66279 & 5.55157 & 6.07341 & 12.49104 & 29.14244 \\
3 & 5.61770 & 10.99370 & 15.93748 & 24.73582 & 35.11061 \\
4 & 7.87228 & 13.35196 & 29.32022 & 30.04191 & 49.20175 \\
5 & 11.80246 & 23.47643 & 30.64494 & 52.82196 & 73.76539 \\
6 & 13.34751 & 30.02651 & 40.44186 & 67.55964 & 83.42192 \\
7 & 21.13354 & 32.91008 & 42.17589 & 74.04768 & 132.08461 \\
8 & 22.03992 & 35.47088 & 70.36587 & 79.80949 & 137.74953 \\
9 & 30.09736 & 43.39231 & 73.55692 & 97.63271 & 188.10852
\end{tabular}

Table 17

Bicubic B-spline solutions of frequency parameters for GFFF plates

\begin{tabular}{cccccr} 
Mode & \multicolumn{5}{c}{ Aspect ratio $r=\mathrm{a} / \mathrm{b}$} \\
sequence & \multicolumn{1}{c}{$2 / 5$} & \multicolumn{1}{c}{$2 / 3$} & 1 & \multicolumn{1}{c}{$3 / 2$} & \multicolumn{1}{c}{$5 / 2$} \\
1 & 3.40206 & 5.34374 & 5.37730 & 5.37070 & 5.35875 \\
2 & 5.60836 & 9.91575 & 14.63677 & 20.81380 & 29.24565 \\
3 & 7.61444 & 10.61721 & 22.02883 & 29.37640 & 33.31023 \\
4 & 9.87651 & 21.63366 & 29.75201 & 50.06930 & 72.76770 \\
5 & 12.89197 & 27.08493 & 36.08762 & 50.25363 & 72.96237 \\
6 & 19.17776 & 30.00296 & 40.16609 & 64.59443 & 124.18050 \\
7 & 20.14829 & 34.78236 & 61.35288 & 74.25586 & 133.93105 \\
8 & 29.46422 & 39.62941 & 66.93716 & 95.03981 & 141.18779 \\
9 & 30.49951 & 48.55998 & 74.01804 & 101.69672 & 155.95143
\end{tabular}

The rate of convergence of the frequencies corresponding to the first six modes is presented in Table 1 . The number of spline sections in both $x$ and $y$ directions is varied from $6 \times 6$ to $20 \times 20$. It can be observed from Table 1 that the rate of convergence for the fundamental frequency is very fast. Full convergence is achieved with $N \times M=$ $14 \times 14$. Based on the convergence study, $14 \times 14$ spline sections are used hereafter for calculating frequencies.

\subsection{Numerical examples}

Bert and Malik [2] presented analytical solutions of frequency parameters for 21 cases of rectangular plates with one or more guided edges. The 21 cases have been sorted into three groups, i.e. plates with two opposite edges simply supported, plates with one edge simply supported and opposite edge guided, and plates with two opposite edges guided. The first nine frequency parameters of three cases from the three groups, i.e. SCSG, SGGF, and GGGG, are computed by the present method and compared with the analytical solutions in Tables $2-4$, respectively. The agreement between the present solutions and analytical ones is impressive. 


\subsection{Bicubic B-spline solutions for plates with guided edges}

As has been stated in the introduction, for rectangular plates with at least one guided edge there exist 34 combinations of edge conditions, among which 21 cases have analytical solutions and have been given by Bert and Malik [2]. However, no study has been reported for the remaining 13 cases that have approximate or numerical solutions only. Nevertheless, the numerical accuracy and versatility of the present bicubic B-spline approach have been well validated from the above comparisons. Bicubic B-spline solutions for the first nine frequency parameters of the 13 cases of rectangular plate over a range of aspect ratios are given in Tables 5-17 for future comparison. The plate geometrical description is the same as shown in Fig. 3 and the aspect ratio $r=a / b$. The frequency parameter is $\omega a^{2} \sqrt{ } \rho / D$.

\section{Conclusions}

This work concerns the free vibration of thin isotropic rectangular plates with at least one guided edge. A versatile type of basis cubic B-spline functions has been first developed to satisfy simply supported, clamped, free, and guided edge conditions with easy numerical manipulation. Incorporating the new type of basis cubic B-spline functions, bicubic B-spline interpolation is used to represent the plate deflection. The frequency characteristic equation is derived on classical thin plate theory by performing Hamilton's principle.

There exist 34 combinations of edge conditions for rectangular plates, with at least one guided edge, among which 21 cases have analytical solutions and have been given by Bert and Malik [2]. The present solutions are compared with the analytical ones by Bert and Malik [2]. Rapid convergence, high accuracy and computational efficiency of the present method have been demonstrated from the excellent agreement of the comparisons.

By using the present bicubic B-spline approach, extensive computations were performed for natural frequencies of 13 cases of rectangular plates having one or more guided edges with various aspect ratios. These numerical results provided in this paper are believed to fill the gap in literature on rectangular plates with guided edge(s).

\section{Appendix}

The elements of the spline matrices in Eqs (27)-(30), i.e. $\left[A_{x}\right],\left[B_{x}\right],\left[C_{x}\right]$ and $\left[F_{x}\right]$, are as following. The dimension of the spline matrices is $(N+3) \times(N+3)$. The spline matrices $\left[A_{y}\right],\left[B_{y}\right],\left[C_{y}\right]$ and $\left[F_{y}\right]$ are in similar form as $\left[A_{x}\right],\left[B_{x}\right],\left[C_{x}\right]$ and $\left[F_{x}\right]$, and the dimension is $(M+3) \times(M+3)$.

$$
\begin{aligned}
& {\left[A_{x}\right]=\begin{array}{c}
1 \\
16 h_{x}^{3}
\end{array}\left[\begin{array}{lrrrrrrr}
36 & 54 & 15 & -48 & 6 & 6 & 0 & 0 \\
& 96 & 27 & -60 & 0 & 6 & 0 & 0 \\
& & 18 & -16 & -1 & 2 & 0 & 0 \\
& & & 96 & 54 & 0 & 6 & 0 \\
\text { Sym. } & & & & \ddots & \ddots & \ddots & \ddots
\end{array}\right]_{(N+3) \times(N+3)}} \\
& {\left[B_{x}\right]=\begin{array}{c}
1 \\
720 h_{x}
\end{array}\left[\begin{array}{rrrrrrrr}
-396 & -954 & -57 & 240 & 150 & 6 & 0 & 0 \\
-234 & -624 & -165 & 84 & 144 & 6 & 0 & 0 \\
& & -54 & 8 & 47 & 2 & 0 & 0 \\
& & & -480 & 90 & 144 & 6 & 0 \\
\text { Sym. } & & & & \ddots & \ddots & \ddots & \ddots
\end{array}\right]_{(N+3) \times(N+3)}} \\
& {\left[C_{x}\right]={ }_{720 h_{x}}\left[\begin{array}{rrrrrrrr}
396 & 234 & 57 & -240 & -150 & -6 & 0 & 0 \\
& 624 & 165 & -84 & -144 & -6 & 0 & 0 \\
& & 54 & -8 & -47 & -2 & 0 & 0 \\
& & & 480 & -90 & -144 & -6 & 0 \\
\text { Sym. } & & & & \ddots & \ddots & \ddots & \ddots
\end{array}\right]_{(N+3) \times(N+3)}}
\end{aligned}
$$




$$
\left[F_{x}\right]=\begin{gathered}
h_{x} \\
30240
\end{gathered}\left[\begin{array}{rrrrrrrr}
36756 & 19854 & 5055 & 7872 & 726 & 6 & 0 & 0 \\
& 13776 & 3819 & 7140 & 720 & 6 & 0 & 0 \\
& & 1098 & 2264 & 239 & 2 & 0 & 0 \\
& & & 14496 & 7146 & 720 & 6 & 0 \\
\text { Sym. } & & & & \ddots & \ddots & \ddots & \ddots
\end{array}\right]_{(N+3) \times(N+3)}
$$

\section{References}

[1] H. Antes, Bicubic fundamental splines in plate bending, International Journal for Numerical Methods in Engineering, 8 (1974), 503-511.

[2] C.W. Bert and M. Malik, Frequency equations and modes of free vibrations of rectangular plates with various edge conditions, Proc. Inst. Mech. Eng., Part C: J. Mech. Eng. Sci. 208C (1994), 307-319.

[3] C.D. Boor, Bicubic spline interpolation, Journal of Mathematics and Physics XLI (1962), 212-218.

[4] C.D. Boor, A practical guide to splines, (Vol. 27), (Applied mathematical sciences, Springer-Verlag, New York, 1978.

[5] J.W. Duan and P.K.K. Lee, Construction of boundary B-spline functions, Computers and Structures 78 (2000), 737-743.

[6] A.W. Leissa, The free vibration of rectangular plates, Journal of Sound and Vibration 31(3) (1973), 257-293.

[7] R. Qin, Spline functions in structural mechanics, Guangxi People Press, China, 1985, pp. 57-64, in Chinese.

[8] L.L. Schumaker, Spline functions: basic theory, John Wiley and Sons, New York, 1981.

[9] P.C. Shen, P.X. He and Y.X. Le, Vibration analysis of plates using the multivariable spline element method, International Journal of Solid and Structures 29(24) (1992), 3289-3295.

[10] P.C. Shen and H.B. Kan, The multivariable spline element analysis for plate bending problems, Computers and Structures 40(6) (1992), 1343-1349.

[11] A.C. Ugural, Stresses in plates and shells, (2nd ed.), McGraw-Hill, Singapore, 1999, pp. 84-87.

[12] G. Wang, and C.T. Hsu, Static and dynamic analysis of arbitrary quadrilateral flexural plates by B-spline functions, International Journal of Solid and Structures 31(5) (1994), 657-667.

[13] D. Young, Vibration of rectangular plates by the Ritz method, Journal of Applied Mechanics, ASME 12 (1950), 448-453. 

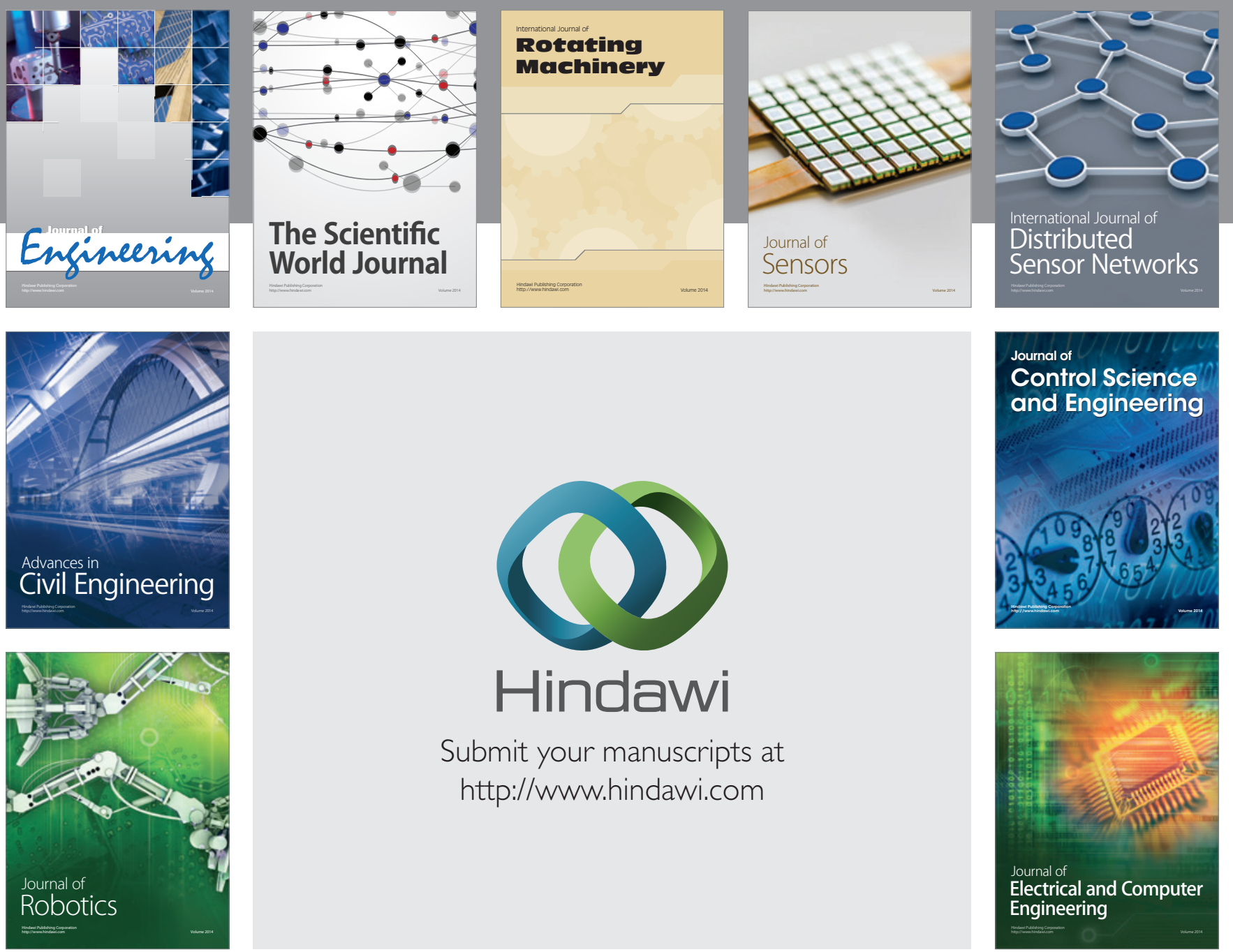

Submit your manuscripts at

http://www.hindawi.com
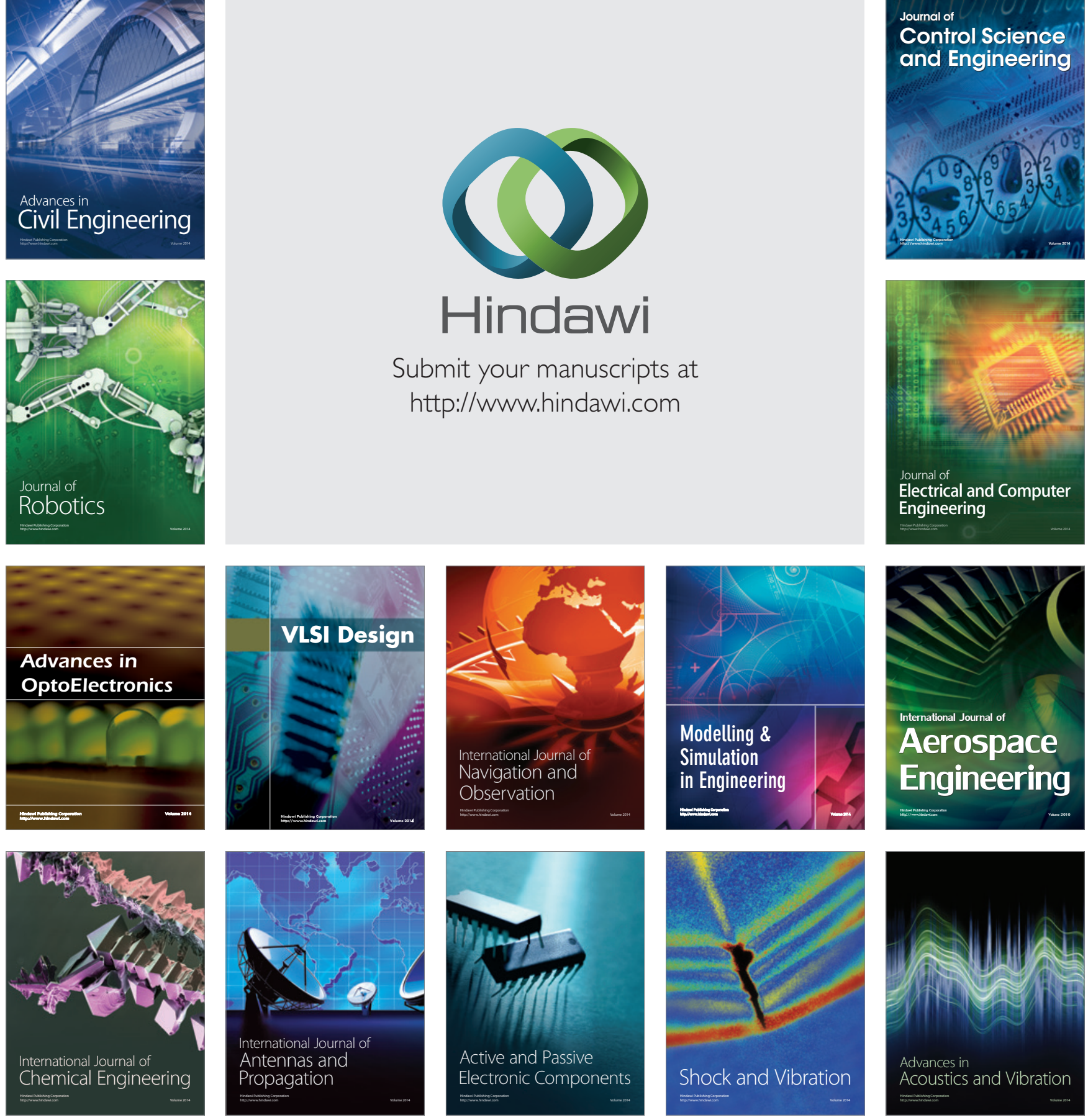